\title{
Climbing plants of Porto Ferreira State Park, southeastern Brazil
}

\author{
Betânia da Cunha Vargas ${ }^{* 1 ; 2}$; Ana Paula Caldeira Oliveira ${ }^{1}$; Renata Giassi Udulutsch ${ }^{3}$; \\ Gabriel Mendes Marcusso ${ }^{1}$; Gabriel Pavan Sabino ${ }^{1}$; Pablo Hendrigo Alves de Melo ${ }^{1}$; \\ Roberta Marotti Martelletti Grillo ${ }^{1}$; Vitor de Andrade Kamimura $^{1}$; Marco Antonio Assis ${ }^{1}$ \\ ${ }^{1}$ Universidade Estadual Paulista, Instituto de Biociências de Rio Claro, Departamento de Botânica, Rio Claro, SP, Brasil \\ ${ }^{2}$ Universidade Estadual Paulista, Instituto de Biociências de Rio Claro, Departamento de Botânica, \\ Laboratório de Fenologia, Rio Claro, SP, Brasil \\ ${ }^{3}$ Universidade Estadual Paulista, Faculdade de Ciências e Letras, Departamento de Ciências Biológicas, Assis, SP, Brasil \\ *Corresponding author: Betânia da Cunha Vargas, e-mail: betaniadacunhavargas@gmail.com
}

VARGAS, B. C.; OLIVEIRA, A. P. C.; UDULUTSCH, R. G.; MARCUSSO, G. M.; SABINO, G. P.; MELO, P. H. A.; GRILLO, R. M. M.; KAMIMURA, V. A.; ASSIS, M. A. Climbing plants of Porto Ferreira State Park, southeastern Brazil. Biota Neotropica. 18(2): e20170346. http://dx.doi.org/10.1590/1676-0611-BN-2017-0346

\begin{abstract}
A floristic survey of climbing plants was carried out in an ecotone area of seasonal semideciduous forest (SSF) and forested savanna (CER), in Porto Ferreira State Park (PFSP), Southeastern Brazil. We sampled the reproductive specimens every month during two periods, March 2010 to September 2011 and April and July 2015. The surveys were performed by the walking method, and the sampled individuals were classified by habit, climbing mechanism and dispersal mode. Overall, 109 species, belonging to 67 genera and 29 families, were recorded; 49 species occurred in both, 29 and 31 were exclusive to SSF and CER, respectively. Bignoniaceae and Malpighiaceae were the richest families, with 17 species, followed by Sapindaceae (12 species), Asteraceae and Apocynaceae ( 8 species each) and Fabaceae (6). The majority of climbers were lianas, twining and anemochoric species, corresponding to $70 \%, 47 \%$ and $66 \%$ of all samples, respectively. In this work, we added one new family and 14 species to the Cerrado's list of climbing plants from São Paulo state, and 10 species to the Brazilian seasonal semideciduous forest's list. Therefore, we contributed to the understanding of diversity of climbing plants in vegetation types poorly studied for this plant group, mainly in the Cerradão, wherein we found new records for several species.
\end{abstract}

Key words: Cerrado; Seasonal Semideciduous Forest; ecotone; climbing plants.

\section{Trepadeiras do Parque Estadual de Porto Ferreira, Brasil}

\begin{abstract}
Resumo: O levantamento florístico das trepadeiras foi realizado em um ecótono de Floresta Estacional Semidecidual (FES) e Cerradão (CER), no Parque Estadual de Porto Ferreira, Sudeste do Brasil. Realizamos coletas mensais dos espécimes reprodutivos ao longo de dois períodos, março 2010 a setembro 2011, abril e julho 2015. Os levantamentos foram realizados por meio do método de caminhada e os indivíduos amostrados foram classificados quanto ao hábito, mecanismo de ascensão e síndrome de dispersão. No geral, foram registradas 109 espécies, pertencentes a 67 gêneros e 29 famílias. Dentre essas, 49 espécies ocorrem nos dois tipos de vegetação, sendo que 29 e 31 espécies são exclusivas de FES e CER, respectivamente. Bignoniaceae e Malpighiaceae foram as famílias mais ricas com 17 espécies, seguidas por Sapindaceae (12 espécies), Asteraceae e Apocynaceae (8 espécies cada) e Fabaceae (6). A maioria das espécies de trepadeiras são lianas, volúveis e anemocóricas, correspondendo a 70\%, 47\% e $66 \%$ de toda a amostra, respectivamente. Neste trabalho, acrescentamos uma nova família e 14 espécies para a lista de trepadeiras do Cerrado paulista e 10 espécies para a lista brasileira de trepadeiras em Floresta Estacional Semidecidual. Portanto, contribuímos para o conhecimento da diversidade de trepadeiras em tipos vegetacionais pouco estudados para este grupo de planta, destacando o Cerradão, no qual encontramos novos registros para um grande número de espécies.
\end{abstract}

Palavras-chave: Cerrado, Floresta Estacional Semidecidual; ecótono; trepadeiras.

\section{Introduction}

Climbing plants germinate and remain on the ground, requiring external mechanical sustentation provided by others plants for access to light in the forest canopy (Darwin 1867; Putz \& Windsor 1987; Gerwing et al. 2006;
Lawdig \& Meiners 2010; van der Heijden et al. 2013). These plants occur in many climates and vegetation types, representing up to $25 \%$ of plant diversity in some tropical forests (Gentry 1991; Engel et al. 1998; Pérez Salicrup et al. 2001). Besides climbing plants playing an important role in biodiversity maintenance, they establish competition for resources with the 
host tree (phorophyte), and can change dynamic and natural regeneration in different biomes (Laurence et al. 2001; Schnitzer \& Bongers 2002).

Although floristic and structural surveys of climbing plants have increased over the past decades (e.g. Durigon \& Waechter 2011; Yorke et al. 2013; Vargas et al. 2014; Ibarra-Marínquez et al. 2015), climbing plants receive less attention in contrast to studies carried out with tree communities (Phillips et al. 2002; Letcher \& Chazdon 2009; Carvalho et al. 2013). In Southeastern Brazil, climbing plant studies have been conducted by floristic and phytosociological surveys, mostly in semideciduous seasonal forest (e.g. Morellato \& Leitão-Filho 1998; Hora \& Soares 2002; Udulutsch et al. 2004; Rezende \& Ranga 2005; Tibiriçá et al. 2006; Rezende et al. 2007; Santos et al. 2009; Udulutsch et al. 2010). Nevertheless, studies on vegetation types of the Cerrado are still incipient (Weiser 2002; 2007; Carvalho et al. 2013).

Porto Ferreira State Park has been the subject of numerous floristic surveys (Bertoni \& Martins 1987; Bertoni et al. 2001; Colli et al. 2003; Oliveira 2012; Osaco 2012; Sabino 2013; Marcusso et al. 2016); however, studies on climbing plants have not been performed in this area. The main objective of this study was to survey the climbing flora of an ecotone area, between seasonal semideciduous forest and forested savanna (Cerradão), in Porto Ferreira State Park, São Paulo, Southeastern Brazil.

\section{Materials and methods}

\section{Study site}

Porto Ferreira State Park (PFSP) is located in the municipality of Porto Ferreira, in São Paulo state, Southeastern Brazil (Figure 1). The study area is 611.55 hectares, and has altitudinal variation between 540 and 608 m.a.s.1. (São Paulo 2003). PFSP is located in a geomorphological region of peripheral depression, in the central region of the watershed of Mogi-Guaçu River (São Paulo 2003).

The climate of the region is classified as Aw, according to Köeppen's classification (Bertoni \& Martins 1987). The annual mean temperature is $22.3^{\circ} \mathrm{C}$ and the annual mean precipitation is $1.497 \mathrm{~mm}$, presenting variation between the rainy $(247.9 \mathrm{~mm})$ and dry seasons $(26.6 \mathrm{~mm})$ (CEPAGRI 2016).

PFSP has distinct vegetation types according to IBGE (2012), presenting forested savanna (Cerradão) and seasonal semideciduous forest. They are mainly differentiated in the field by their physiognomy (small average height and high density in the Cerradão), and greater average height and lower density in Semideciduous forests), ecological aspects (soil types, deciduousness) and typical and exclusive species of each vegetation type (Durigan et al. 2012). Furthermore, there are ecotonal areas among these vegetation types (Osaco 2012; Sabino 2013), in which a floristic mixture and phytophysiognomic indistinguishability occurs (IBGE 2012).
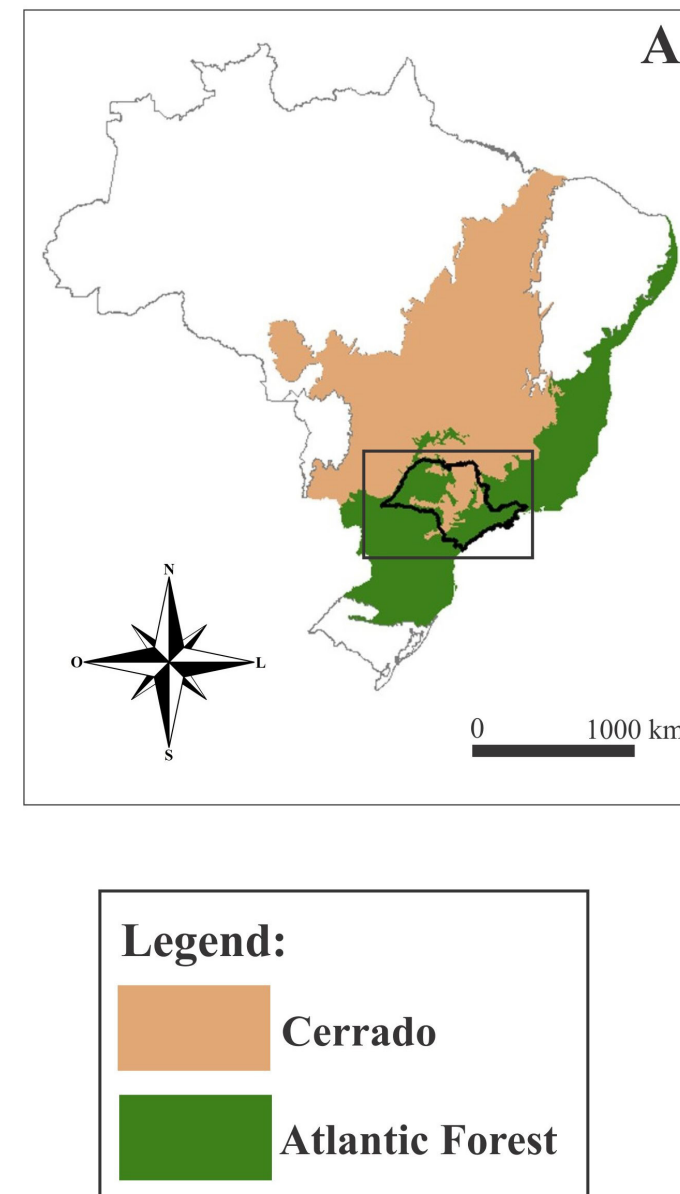

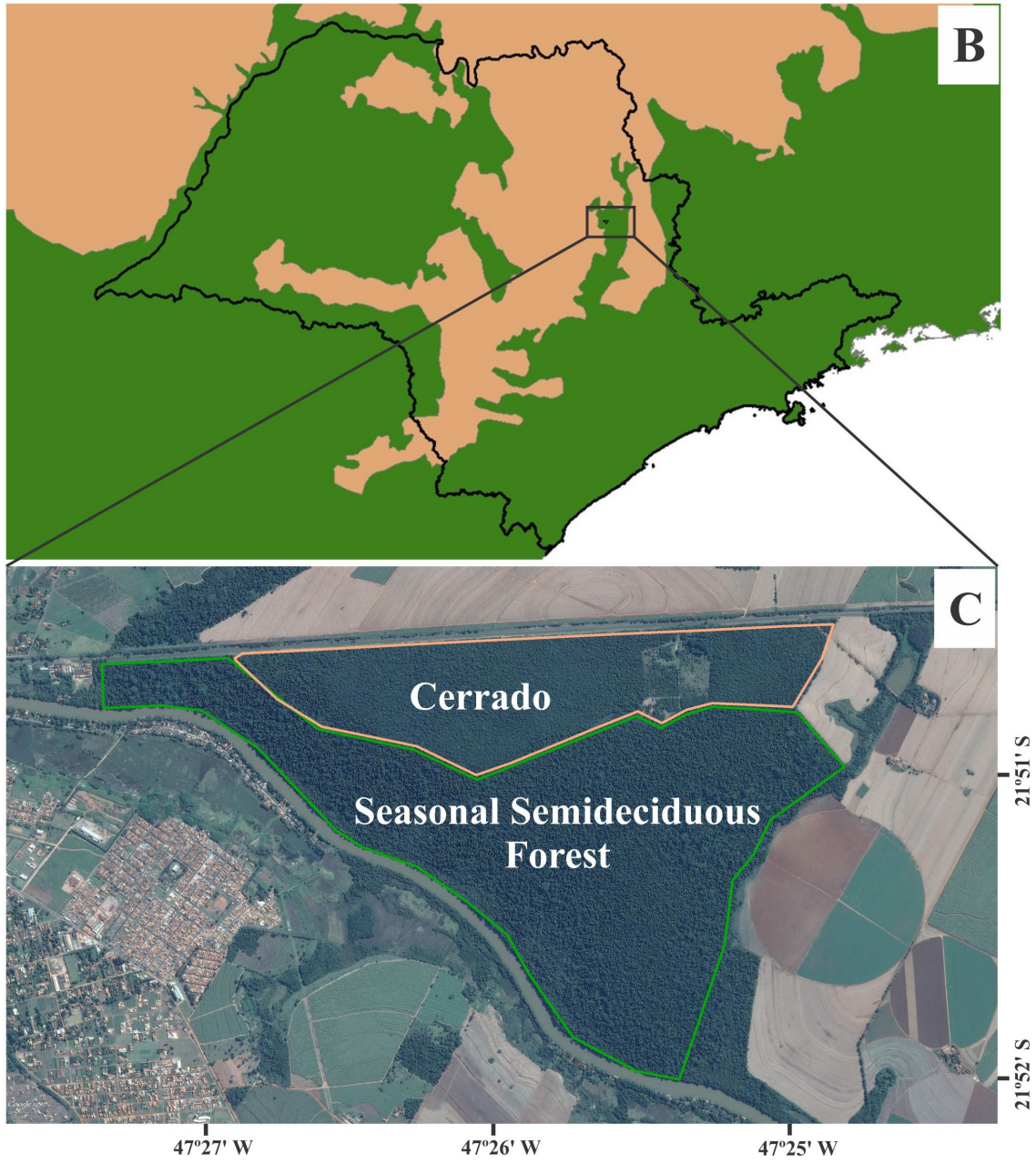

Figure 1. A. Brazilian Atlantic Forest and the Cerrado and the location of São Paulo state; B. location of Porto Ferreira State Park; C. Porto Ferreira State Park and the main vegetation types studied. 


\subsection{Data collection}

Climbing plants sampling was performed by the walking method (Filgueiras et al. 1994), every month between March 2010 and September 2011. According to Morellato et al. (1996), transitional and final periods between climatic seasons can be related to the flowering and fruiting peaks of climbing plants, especially of the families most abundant in species. In this way, aiming to improve the sample, we performed additional floristic surveys in April and July 2015. The reproductive specimens collected in the field were processed and herborized following the protocols of Fidalgo \& Bononi (1984). The vouchers were incorporated in the Herbarium Rioclarense (HRCB). Taxonomic identity was determined through specialized literature, direct comparison with identified specimens in HRCB's collection and by expert taxonomists (cited in the acknowledgements). Synonyms were verified by BFG (2015), and we adopted APG IV (2016) to classify the botanical families.

Identified plants were classified into two different habits: woody (lianas - plants with a secondary growth stem) or herbaceous (plants without a secondary growth stem) climbing plants, following the protocols of Gentry (1991) and of Villagra \& Romaniuc Neto (2014). Climbing mechanisms were categorized as twining, tendril climbing or scandent (Hergaty 1991; Villagra \& Romaniuc Neto 2014). Additionally, we classified climbing plants according to their dispersal modes: anemochory, autochory or zoochory (van der Pijl 1982; Barroso et al. 1999; Peres 2016). We compared the species sampled here with the list of climbing plants for seasonal semideciduous forest (Santos et al. 2009), savanna (Weiser \& Martins 2014) and Brazilian flora (BFG 2015).

\section{Results}

We recorded 109 species, distributed in 67 genera and 29 families (Table 1). We found 78 and 80 species in Seasonal Semideciduous Forest and Cerradão, respectively, of which 49 (45\%) occurred in both, and $29(26.6 \%)$ and $31(28.4 \%)$ were exclusive for the specific vegetation types. The richest families were Bignoniaceae and Malpighiaceae (17 species each), followed by Sapindaceae (12), Asteraceae and Apocynaceae ( 8 species each) and Fabaceae (6), corresponding to $46.7 \%$ of overall richness (Figure 2). In contrast, 11 families ( $38 \%$ of total families) had only one species recorded.

Among the habits, 76 species (70\%) were lianas (woody climbers). The three richest families were entirely composed by woody plants (Table 1). The twining climbing mode was the most representative strategy (51 species; 47\%), followed by tendril climbing (37 species, 37\%) and scandent (18 species, 16\%).

Anemochory was the most representative dispersal mechanism among the climbing plants species surveyed (72 species; $66 \%$ ), followed by zoochory (23 species, $21 \%$ ) and autochory (14 species, 13\%). Among lianas, anemochory was the main dispersal mode (63 species, 83\%), followed by zoochory ( 8 species, $10.5 \%$ ) and autochory (5 species, $6.5 \%$ ), while among herbaceous species, zoochory was the most frequent dispersal mode ( 15 species, $45.4 \%$ ), followed by autochory and anemochory (9 species each, 27.3\%).

In this study, we have registered one new family and 14 species for the Cerrado list, and 10 species for the seasonal semideciduous forest list (Table 1).

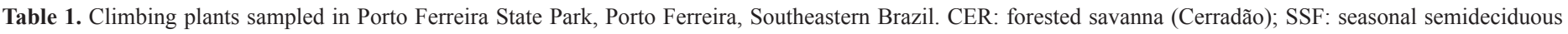

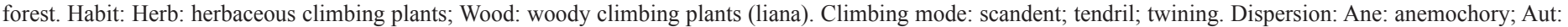

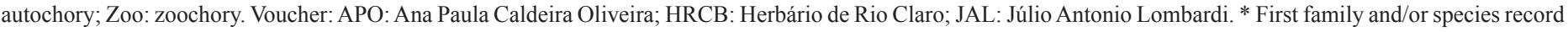
for FES; • first record of the family and/or species for the Cerrado (Santos et al. 2009; Weiser \& Martins 2014; Brazilian Flora Group 2015).

\begin{tabular}{|c|c|c|c|c|c|}
\hline Family/species & Vegetation type & Habit & Climbing mode & Dispersion & Voucher \\
\hline \multicolumn{6}{|l|}{ Acanthaceae } \\
\hline Mendoncia puberula Mart. & SSF & Herb. & Twining & Zoo. & JAL 7712 \\
\hline \multicolumn{6}{|l|}{ Amaranthaceae } \\
\hline Alternanthera brasiliana (L.) Kuntze & $\mathrm{SSF}^{*}$ & Herb. & Scandent & Ane. & JAL 7710 \\
\hline Chamissoa acuminata Mart. & $\mathrm{SSF}^{*}$ & Herb. & Scandent & Ane. & HRCB 68052 \\
\hline \multicolumn{6}{|l|}{ Apocynaceae } \\
\hline Condylocarpon isthmicum (Vell.) A.DC. & $\mathrm{CER}^{\bullet}, \mathrm{SSF}$ & Wood & Twining & Ane. & JAL 7763 \\
\hline Forsteronia australis Müll.Arg. & CER & Wood & Twining & Ane. & HRCB 67992 \\
\hline Forsteronia pubescens A.DC. & CER, SSF & Wood & Twining & Ane. & HRCB 67993 \\
\hline Odontadenia lutea (Vell.) Markgr. & CER & Wood & Twining & Ane. & HRCB 68001 \\
\hline Oxypetalum appendiculatum Mart. & CER & Herb. & Twining & Ane. & HRCB 67994 \\
\hline Prestonia coalita (Vell.) Woodson & CER, SSF & Wood & Twining & Ane. & HRCB 67995 \\
\hline Secondatia densiflora A.DC. & CER, SSF & Wood & Twining & Ane. & HRCB 67996 \\
\hline Temnadenia violacea (Vell.) Miers & CER, SSF & Wood & Twining & Ane. & HRCB 68066 \\
\hline \multicolumn{6}{|l|}{ Aristolochiaceae } \\
\hline Aristolochia labiata Willd. & CER, SSF & Wood & Twining & Aut. & HRCB 67998 \\
\hline \multicolumn{6}{|l|}{ Asteraceae } \\
\hline Bidens segetum Mart. ex Colla & CER, SSF & Wood & Scandent & Ane. & HRCB 67999 \\
\hline Cyrtocymura scorpioides (Lam.) H.Rob. & SSF & Herb. & Scandent & Ane. & APO 116 \\
\hline Dasyphyllum brasiliense (Spreng.) Cabrera & SSF & Wood & Scandent & Ane. & HRCB 68000 \\
\hline Mikania cordifolia (L. f.) Willd. & CER & Herb. & Twining & Ane. & APO 7 \\
\hline Mikania laevigata Sch.Bip. ex Baker & CER & Wood & Scandent & Ane. & APO 110 \\
\hline Mikania sp. 1 & SSF & Wood & Twining & Ane. & APO 117 \\
\hline Mikania sp. 2 & SSF & Wood & Twining & Ane. & APO 104 \\
\hline Mikania triangularis Baker & SSF & Herb. & Twining & Ane. & APO 103 \\
\hline
\end{tabular}


Table 1. Continued...

\begin{tabular}{|c|c|c|c|c|c|}
\hline Family/species & Vegetation type & Habit & Climbing mode & Dispersion & Voucher \\
\hline \multicolumn{6}{|l|}{ Bignoniaceae } \\
\hline Adenocalymma bracteatum (Cham.) DC. & CER, SSF & Wood & Tendril & Ane. & JAL 7730 \\
\hline Adenocalymma marginatum (Cham.) DC. & $\mathrm{CER}^{\bullet}, \mathrm{SSF}$ & Wood & Tendril & Ane. & JAL 7784 \\
\hline Amphilophium crucigerum (L.) L.G.Lohmann & CER, SSF & Wood & Tendril & Ane. & HRCB 68002 \\
\hline Amphilophium elongatum (Vahl) L.G. Lohmann & CER, SSF & Wood & Tendril & Ane. & HRCB 68003 \\
\hline Anemopaegma chamberlaynii (Sims) Bureau \& K. Schum. & CER, SSF & Wood & Tendril & Ane. & JAL 7745 \\
\hline Bignonia campanulata Cham. & $\mathrm{CER}^{\bullet}, \mathrm{SSF}$ & Wood & Tendril & Ane. & HRCB 68006 \\
\hline Cuspidaria convoluta (Vell.) A.H.Gentry & CER, SSF & Wood & Tendril & Ane. & HRCB 68007 \\
\hline Cuspidaria pulchra (Cham.) L.G.Lohmann & CER, SSF & Wood & Tendril & Ane. & JAL 7765 \\
\hline Dolichandra unguis-cati (L.) L.G. Lohmann & SSF & Wood & Tendril & Ane. & HRCB 68009 \\
\hline Fridericia craterophora (DC.) L.G.Lohmann & CER & Wood & Tendril & Ane. & HRCB 68005 \\
\hline Fridericia florida (DC.) L.G.Lohmann & CER & Wood & Tendril & Ane. & HRCB 68004 \\
\hline Fridericia formosa (Bureau) L.G. Lohmann & CER, SSF & Wood & Tendril & Ane. & HRCB 68010 \\
\hline Fridericia speciosa Mart. & CER, SSF & Wood & Tendril & Ane. & HRCB 68061 \\
\hline Lundia obliqua Sond. & $\mathrm{CER}^{\bullet}, \mathrm{SSF}$ & Wood & Tendril & Ane. & APO 62 \\
\hline Pyrostegia venusta (Ker-Gawl.) Miers & CER, SSF & Wood & Tendril & Ane. & HRCB 68060 \\
\hline Tanaecium selloi (Spreng.) L.G. Lohmann & CER, SSF & Wood & Tendril & Ane. & APO 40 \\
\hline Stizophyllum perforatum (Cham.) Miers & CER, SSF & Wood & Tendril & Ane. & HRCB 68012 \\
\hline \multicolumn{6}{|l|}{ Boraginaceae } \\
\hline Myriopus rubicundus (Salzm. ex DC.) Luebert & $\mathrm{CER}^{\bullet}$ & Wood & Scandent & Zoo. & HRCB 68013 \\
\hline Varronia urticifolia (Cham.) J.S.Mill. & $\mathrm{CER}^{\bullet}$ & Wood & Scandent & Zoo. & HRCB 68014 \\
\hline \multicolumn{6}{|l|}{ Cactaceae } \\
\hline Pereskia aculeata Mill. & SSF & Wood & Scandent & Zoo. & JAL 8087 \\
\hline \multicolumn{6}{|l|}{ Cannabaceae } \\
\hline Celtis iguanaea (Jacq.) Sarg. & SSF & Wood & Scandent & Zoo. & JAL 8532 \\
\hline \multicolumn{6}{|l|}{ Celastraceae } \\
\hline Semialarium paniculatum (Mart. ex Schult.) N.Hallé & SSF & Wood & Scandent & Ane. & JAL 8534 \\
\hline \multicolumn{6}{|l|}{ Commelinaceae } \\
\hline Dichorisandra hexandra (Aubl.) C.B.Clarke & SSF & Herb. & Scandent & Zoo. & JAL 7671 \\
\hline \multicolumn{6}{|l|}{ Convolvulaceae } \\
\hline Ipomoea chondrosepala Hallier f. & $\mathrm{CER}, \mathrm{SSF}^{*}$ & Herb. & Twining & Aut. & JAL 7743 \\
\hline Ipomoea saopaulista O’Donell & CER, SSF & Herb. & Twining & Aut. & JAL 7744 \\
\hline Merremia macrocalyx (Ruiz \& Pav.) O’Donell & CER, SSF & Herb. & Twining & Aut. & HRCB 68015 \\
\hline Merremia umbellata (L.) Hallier f. & $\mathrm{CER}, \mathrm{SSF}^{*}$ & Herb. & Twining & Aut. & APO 60 \\
\hline \multicolumn{6}{|l|}{ Cucurbitaceae } \\
\hline Gurania sp. & $\mathrm{CER}^{\bullet}, \mathrm{SSF}^{*}$ & Herb. & Tendril & Zoo. & APO 55 \\
\hline Psiguria ternata (M.Roem.) C.Jeffrey & CER & Herb. & Tendril & Zoo. & HRCB 68016 \\
\hline \multicolumn{6}{|l|}{ Cyperaceae } \\
\hline Scleria latifolia Sw. & $\mathrm{SSF}$ & Herb. & Scandent/thorn & Zoo. & HRCB 68059 \\
\hline \multicolumn{6}{|l|}{ Dilleniaceae } \\
\hline Davilla elliptica A.St.-Hil. & $\mathrm{SSF}^{*}$ & Wood & Twining & Zoo. & HRCB 68017 \\
\hline Davilla rugosa Poir. & CER, SSF & Wood & Twining & Zoo. & APO 46 \\
\hline Doliocarpus dentatus (Aubl.) Standl. & CER, SSF & Wood & Twining & Zoo. & APO 21 \\
\hline \multicolumn{6}{|l|}{ Dioscoreaceae } \\
\hline Dioscorea dodecaneura Vell. & CER, SSF & Herb. & Twining & Ane. & JAL 7736 \\
\hline Dioscorea olfersiana Klotzsch ex Griseb. & CER, SSF & Herb. & Twining & Ane. & JAL 7741 \\
\hline \multicolumn{6}{|l|}{ Euphorbiaceae } \\
\hline Dalechampia pentaphylla Lam. & $\mathrm{CER}^{\bullet}, \mathrm{SSF}$ & Herb. & Twining & Aut. & JAL 7777 \\
\hline Dalechampia stipulacea Müll.Arg. & SSF & Herb. & Twining & Aut. & JAL 7709 \\
\hline Dalechampia triphylla Lam. & SSF & Herb. & Twining & Aut. & HRCB 68019 \\
\hline \multicolumn{6}{|l|}{ Fabaceae } \\
\hline Canavalia picta Mart. ex Benth. & CER & Wood & Twining & Aut. & APO 92 \\
\hline Centrosema sagittatum (Humb. \& Bonpl. ex Willd.) Brandegee & $\mathrm{SSF}$ & Herb. & Twining & Aut. & HRCB 68020 \\
\hline Dioclea violacea Mart. ex Benth. & $\mathrm{SSF}$ & Wood & Twining & Aut. & HRCB 68021 \\
\hline Rhynchosia minima (L.) DC. & $\mathrm{CER} \bullet \mathrm{SSF}$ & Herb. & Twining & Aut. & JAL 7752 \\
\hline Rhynchosia phaseoloides (Sw.) DC. & SSF & Wood & Twining & Aut. & HRCB 68022 \\
\hline Senegalia polyphylla (DC.) Britton \& Rose & CER & Wood & Scandent/thorn & Aut. & JAL 8531 \\
\hline
\end{tabular}


Table 1. Continued...

\begin{tabular}{|c|c|c|c|c|c|}
\hline Family/species & Vegetation type & Habit & Climbing mode & Dispersion & Voucher \\
\hline \multicolumn{6}{|l|}{ Lygodiaceae } \\
\hline Lygodium volubile Sw. & CER, SSF & Herb. & Twining & Ane. & HRCB 68050 \\
\hline \multicolumn{6}{|l|}{ Malpighiaceae } \\
\hline Banisteriopsis adenopoda (A.Juss.) B.Gates & $\mathrm{CER}^{\bullet}$ & Wood & Twining & Ane. & APO 91 \\
\hline Banisteriopsis argyrophylla (A.Juss.) B.Gates & CER, SSF & Wood & Twining & Ane. & JAL 7755 \\
\hline Banisteriopsis latifolia (A.Juss.) B.Gates & CER & Wood & Twining & Ane. & HRCB 68025 \\
\hline Banisteriopsis malifolia (Nees \& Mart.) B.Gates var. malifolia & CER, SSF & Wood & Twining & Ane. & HRCB 68026 \\
\hline Banisteriopsis cf. muricata (Cav.) Cuatrec. & CER, SSF & Wood & Twining & Ane. & HRCB 68031 \\
\hline Banisteriopsis oxyclada (A.Juss.) B.Gates & CER, SSF & Wood & Twining & Ane. & HRCB 68027 \\
\hline Banisteriopsis stellaris (Griseb.) B.Gates & CER & Wood & Twining & Ane. & HRCB 68028 \\
\hline Banisteriopsis variabilis B.Gates & CER & Wood & Scandent & Ane. & HRCB 68029 \\
\hline Diplopterys pubipetala (A.Juss.) W.R.Anderson \& C.C.Davis & CER & Wood & Twining & Ane. & APO 114 \\
\hline Heteropterys umbellata A.Juss. & $\mathrm{SSF}^{*}$ & Wood & Twining & Ane. & JAL 8089 \\
\hline Heteropterys sp. & CER & Wood & Twining & Ane. & HRCB 68030 \\
\hline Mascagnia cordifolia (A.Juss.) Griseb. & CER & Wood & Twining & Ane. & APO 115 \\
\hline Mascagnia sepium (A.Juss.) Griseb. & CER, SSF & Wood & Twining & Ane. & JAL 7732 \\
\hline Niedenzuella lucida (A.Juss.) W.R.Anderson & $\mathrm{SSF}^{*}$ & Wood & Twining & Ane. & HRCB 68034 \\
\hline Niedenzuella multiglandulosa (A.Juss.) W.R.Anderson & CER, SSF & Wood & Twining & Ane. & HRCB 68032 \\
\hline Stigmaphyllon lalandianum A.Juss. & SSF & Wood & Twining & Ane. & HRCB 68033 \\
\hline Tetrapterys sp. & SSF & Wood & Twining & Ane. & HRCB 68049 \\
\hline \multicolumn{6}{|l|}{ Menispermaceae } \\
\hline Cissampelos pareira $\mathrm{L}$. & CER & Wood & Twining & Zoo. & JAL 8536 \\
\hline \multicolumn{6}{|l|}{ Passifloraceae } \\
\hline Passiflora alata Curtis & $\mathrm{SSF}^{*}$ & Herb. & Tendril & Zoo. & JAL 7767 \\
\hline Passiflora miersii Mast. & CER & Herb. & Tendril & Zoo. & APO 95 \\
\hline Passiflora suberosa L. & CER & Herb. & Tendril & Zoo. & APO 2 \\
\hline \multicolumn{6}{|l|}{ Polygalaceae } \\
\hline Bredemeyera floribunda Willd. & CER, SSF & Wood & Scandent & Ane. & HRCB 68035 \\
\hline Securidaca tomentosa A.St.Hil. \& Moq. & $\mathrm{CER}^{\bullet}$ & Wood & Scandent & Ane. & HRCB 68065 \\
\hline \multicolumn{6}{|l|}{ Ranunculaceae $^{\bullet}$} \\
\hline Clematis dioica $\mathrm{L}$. & $\mathrm{CER}^{\bullet}$ & Wood & Twining & Ane. & JAL 8530 \\
\hline \multicolumn{6}{|l|}{ Rhamnaceae } \\
\hline Gouania virgata Reissek & CER, SSF & Wood & Tendril & Ane. & HRCB 68037 \\
\hline \multicolumn{6}{|l|}{ Rubiaceae } \\
\hline Galianthe laxa (Cham. \& Schltdl.) E.L.Cabral & CER, SSF & Herb. & Scandent & Zoo. & JAL 7738 \\
\hline Manettia cordifolia Mart. & CER, SSF & Herb. & Twining & Zoo. & HRCB 68038 \\
\hline \multicolumn{6}{|l|}{ Sapindaceae } \\
\hline Cardiospermum grandiflorum Sw. & SSF & Wood & Tendril & Ane. & HRCB 68039 \\
\hline Paullinia rhomboidea Radlk. & CER, SSF & Wood & Tendril & Ane. & APO 102 \\
\hline Serjania cf. acoma Radlk. & CER & Wood & Tendril & Ane. & HRCB 68040 \\
\hline Serjania communis Cambess. & $\mathrm{CER}^{\bullet}$ & Wood & Tendril & Ane. & APO 3 \\
\hline Serjania fuscifolia Radlk. & $\mathrm{CER}^{\bullet}, \mathrm{SSF}$ & Wood & Tendril & Ane. & APO 44 \\
\hline Serjania laruotteana Cambess. & CER & Wood & Tendril & Ane. & HRCB 68041 \\
\hline Serjania lethalis A.St.-Hil. & CER & Wood & Tendril & Ane. & APO 107 \\
\hline Serjania meridionalis Cambess. & $\mathrm{SSF}$ & Wood & Tendril & Ane. & JAL 7715 \\
\hline Serjania paradoxa Radlk. & CER & Wood & Tendril & Ane. & APO 120 \\
\hline Serjania pinnatifolia Radlk. & CER, SSF & Wood & Tendril & Ane. & HRCB 68055 \\
\hline Serjania reticulata Cambess. & CER, SSF & Wood & Tendril & Ane. & APO 48 \\
\hline Urvillea laevis Radlk. & CER, SSF & Wood & Tendril & Ane. & APO 63 \\
\hline \multicolumn{6}{|l|}{ Smilaceae } \\
\hline Smilax elastica Griseb. & CER & Herb. & Tendril & Zoo. & HRCB 68043 \\
\hline Smilax fluminensis Steud. & CER & Herb. & Tendril & Zoo. & HRCB 68058 \\
\hline \multicolumn{6}{|l|}{ Vitaceae } \\
\hline Cissus erosa Rich. & CER, SSF & Herb. & Tendril & Zoo. & HRCB 68045 \\
\hline Cissus tinctoria Mart. & SSF & Herb. & Tendril & Zoo. & HRCB 68046 \\
\hline Cissus verticillata (L.) Nicolson \& C.E.Jarvis & SSF & Herb. & Tendril & Zoo. & HRCB 68047 \\
\hline
\end{tabular}




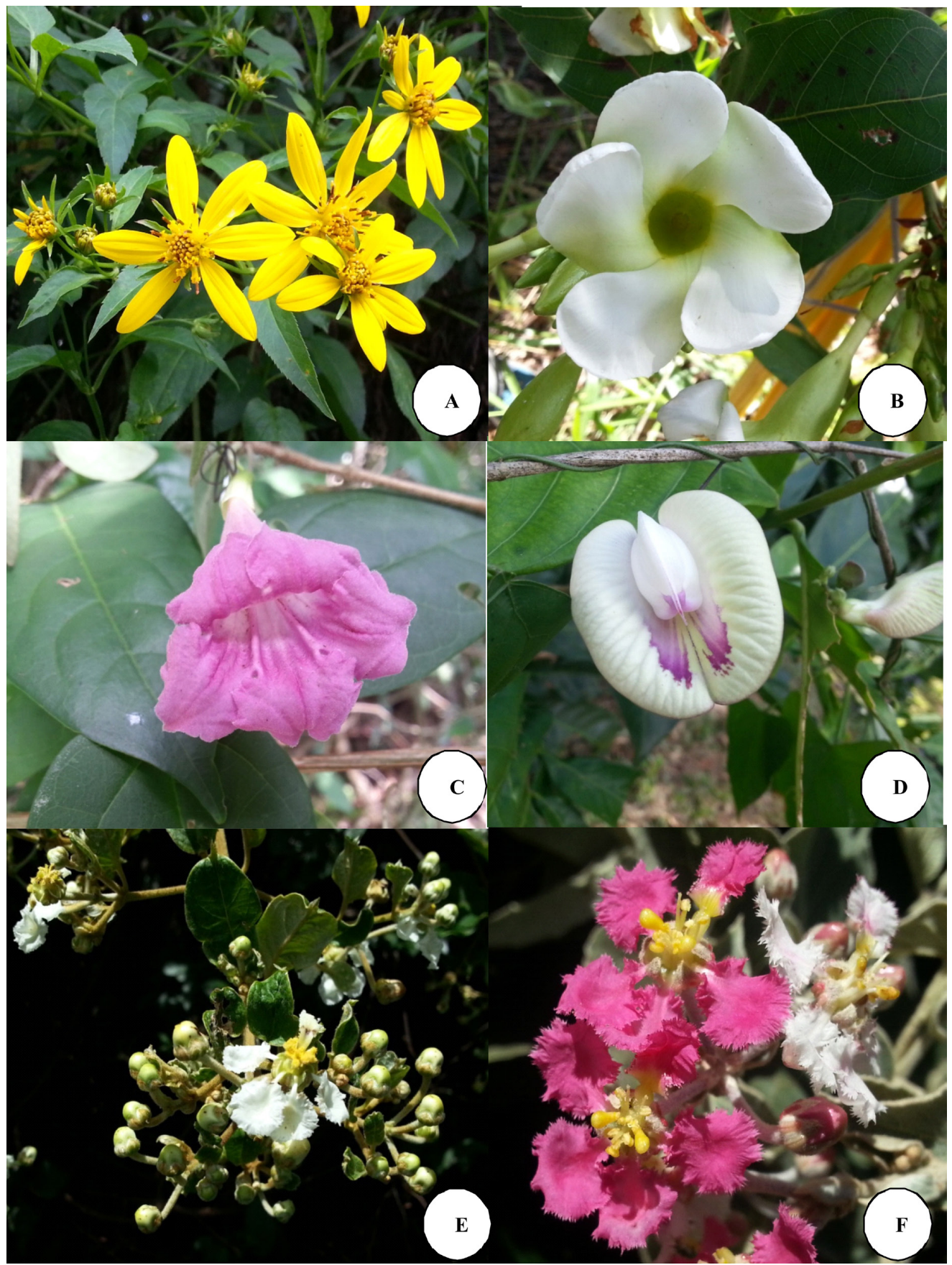

Figure 2. A. Bidens segetum; B. Odontadenia lutea; C. Fridericia craterophora; D. Centrosema sagittatum; E. Banisteriopsis argyrophylla; F. Banisteriopsis adenopoda. By: Marcusso, G., Sabino, G. and Udulutsch, R.G.

\section{Discussion}

The present study showed elevated richness of climbing plants in Cerradão, compared to other studies carried out in Cerrado sensu stricto in São Paulo state, where 41 (Mantovani \& Martins 1993) and 15 species (Weiser \& Godoy 2001) were recorded. On the other hand, in a Cerradão in Bauru, 52 species of climbers were recorded (Weiser 2007), corroborating our results that the Cerradão is richer in climbing plants than Cerrado sensu stricto. Although these studies had different sample time spans, the richness recorded in the present study can be considered high by the fact that the studies carried out in Cerrado sensu stricto had very similar sample times to ours (16 to 22 months, Mantovani \& Martins 1993; Weiser \& Godoy 2001, respectively), while the study in Cerradão considered a much larger sample time (51 months, Weiser 2007). However, the last considered a smaller area than the present study (1 versus 169 hectares, respectively). In this way, it is likely that other Cerradão areas are as rich in this vegetation type as that recorded in the present study.

Otherwise, the number of species found in Seasonal Semideciduous Forest can be considered low compared to the average for this vegetation type (e.g. Morellato \& Leitão-Filho 1998; Udulutsch et al. 2004; Rezende \& Ranga 2005; Tibiriçá et al. 2006; Carneiro \& Vieira 2012). However, we did not sample months with the same frequency, as we collected one more 
time during transitional months and those at the end of the climatic season (rainy and dry season), and these reproductive periods are considered the peak of flowering and fruiting for climbing plants (Morellato \& Leitão-Filho 1996). This was also the case in the study of Udulutsch et al. (2010), so much that these surveys showed a similar number of species (74 species) to the present study ( 78 species). Furthermore, floristic surveys are usually carried out for different time spans and in areas with different sizes, which compromises comparisons of diversity and the relationships between flora of different sites (e.g. Forzza et al. 2014). In this way, we suggest that floristic studies are made considering equal sample frequency during the year, considering the reproductive stages of the plants. Therefore, it may be that the Seasonal Semideciduous Forest of PFSP is so rich as those in other studies which have reported high values for richness (e.g. Morellato \& Leitão- Filho 1998; Udulutsch et al. 2004; Tibiriçá et al. 2006).

In general, the reasonably substantial number of climbing plant species recorded in this study is perhaps associated with the ecotonal condition of this area, where the floristic elements of both vegetation types (seasonal semideciduous forest and Cerradão) contribute to the richness. Although for climbing plants this aspect is poorly known, this has already been documented for vascular epiphytes (e.g. Kersten 2010; Bonnet et al. 2011; Marcusso \& Monteiro 2016) and trees (e.g. Pinheiro \& Monteiro 2008). This elevated richness and abundance of species in an ecotone is related to the meeting of adjacent areas, and thus encompasses the diversity of the community around it (Odum 1969; Kent et al. 1997; Kark 2012). This transitional area shares environmental conditions but also establishes the particular conditions of an ecotone, allowing coexistence of species from the surrounding vegetation, as well as rare and endemic species (Kark \& Rensburg 2006; Kark 2012).

The majority of climbing plants species registered belong to few families, corroborating the hypothesis described by Gentry (1991) in which the majority of species ( $70 \%$ in the present study) are concentrated in only 13 families. Among the richest families, Bignoniaceae, Malpighiaceae and Sapindaceae stand out (e.g. Lombardi et al. 1999; Nabe-Nielsen 2001; Hora \& Soares 2002; Tibiriçá et al. 2006). These families have a widespread geographical distribution, occurring among different vegetation types (Lohmann 2015; Mamede et al. 2015; Somner et al. 2015), and certain genera are predominantly (e.g. Bignoniaceae: Fridericia; Sapindaceae: Serjania; Malpighiaceae: Banisteriopsis) or exclusively (e.g. Bignoniaceae: Lundia, Pyrostegia, Tanaecium, Stizophyllum; Sapindaceae: Urvillea; Malpighiaceae: Mascagnia, Niedenzuella) composed by climbing species (Gentry 1991; BFG 2015).

We found a prevalence of lianas in the study area. Although an equal proportion of lianas and herbaceous climbing plants is expected in tropical forests (Gentry \& Dodson 1987; Gentry 1991; Durigon et al. 2009), studies of climbing plants realized in semideciduous forest found $64 \%$ (Udulutsch et al. 2004) and 60\% (Vargas et al. 2013) lianas, contradicting this estimation. On the other hand, herbaceous vines can represent almost $85 \%$ of the richness in subtropical and temperate forests (Durigon et al. 2014).

The pattern of climbing mechanism registered for tropical forests was corroborated in this study, wherein twining was the predominant mechanism, followed by tendril climbing (e.g. Gentry 1991; Araujo \& Alves 2010; Vivek \& Parthasarathy 2015). Despite some species having specialized structures to assist in colonization of the phorophyte, such as tendrils modified with adhesive pads and hooks, improving their colonization success (Gentry 1979; Lohmann 2006), this climbing mechanism has not been the most encountered in tropical forests (Gentry 1991). In this study, we recorded Scleria latifolia and Senegalia polyphylla with a twining habit. In general, these species are not included in climbing plant floristic surveys, but in PFSP these species were observed with this growth mode; perhaps they use scabrous and thorn features to ascend in the phorophyte.

In general, anemochory ( 72 species) was the most commonly found dispersal mode among climbing plants (lianas + herbaceous vines) (Durigon \& Waechter 2011; Gallagher et al. 2011; Vargas et al. 2013), which is associated with environments with pronounced climatic seasonality (Dewalt et al. 2010; Schnitzer \& Bongers 2011). According to Morellato \& Leitão-Filho (1996), the pattern of dispersion can be considered complementary in forest phenology, wherein in periods with a smaller amount of annual precipitation, the tree community totally or partially loses its leaves (Montovani \& Martins 1998), and climbing plants can use wind currents to disperse their seeds (Morellato \& Leitão-Filho 1998). Although, when considering only herbaceous vines, this relation can be altered by the predominance of zoochory, thus their fruits mature during the rainy season (Morellato \& Leitão-Filho 1996).

Despite floristic inventories of climbing plants increasing, they are concentrated in semideciduous forest (e.g. Hora \& Soares 2002; Tibiriçá et al. 2006; Rezende et al. 2007; Udulutsch et al. 2010; Vargas et al. 2013), to the detriment of those realized in the Cerrado (Weiser \& Godoy 2001; Weiser 2007; Oliveira et al. 2014). However, this study included new registers of species for both vegetation types. This is because climbing plants comprise a very diverse group, so much so that they are considered key to innovation throughout evolution, increasing angiosperm diversity (Gianoli 2015; Gianoli et al. 2016). For this reason, we highlight the importance of including climbing plants in community research (Durigon et al. 2014; Schnitzer et al. 2015), and realizing floristic inventories that include or integrate climbing plants.

The present study reveals the considerable richness of this sinusia in Porto Ferreira State Park, with a similar number of species between the vegetation types. The mainly contribution is relation to diversity, recorded species, like Scleria latifolia and Senegalia polyphylla, as climbing plants. Both to vegetation types showed new records to the climbing plants checklist. Thus, we encourage the initiatives of floristic surveys with climbing plants, with similar frequency of sample, in these vegetation types, mainly in the Cerradão, in view of the importance of its floristic richness and to filling out the remaining gaps.

\section{Acknowledgements}

We would like to thank the following experts, followed by their respective linked herbaria, for help with some identifications: Maria Cândida Henrique Mamede (SP), Rosângela Simão Bianchini (SP), Lidyanne Y. Saleme Aona (UFRB), Luiza Sumiko Kinoshita (UEC), Rubens Teixeira Queiroz (UEC), Ana Maria Goulart de Azevedo Tozzi (UEC), Ana Paula Fortuna-Perez (BOTU), Marcelo Monge Egea (UEC), João Semir (UEC) and Rafael Felipe de Almeida (HUEFS); the employees of PFSP; Capes and CNPq for the fellowship grant; ; and São Paulo Research Foundation (FAPESP) for fellowship grant 2015/13112-7 to P.H.A. Melo. We like to thank to J.A. Lombardi contributions with data collection.

\section{Author Contributions}

Marco Antonio Assis and Renata Giassi Udulutsch: Contribution in the concept and design of the study

Betânia da Cunha Vargas, Renata Giassi Udulutsch, Gabriel Mendes Marcusso, Gabriel Pavan Sabino, Pablo Hendrigo Alves de Melo, Roberta Marotti Martelletti Grillo, Vitor de Andrade Kamimura, Marco Antonio Assis: Contribution to data collection

Betânia da Cunha Vargas, Gabriel Mendes Marcusso, Gabriel Pavan Sabino, Pablo Hendrigo Alves de Melo, Vitor de Andrade Kamimura: Contribution to data analysis and interpretion

Betânia da Cunha Vargas, Gabriel Mendes Marcusso, Gabriel Pavan Sabino, Roberta Marotti Martelletti Grillo, Vitor de Andrade Kamimura: Contribution to manuscript preparation

Betânia da Cunha Vargas, Renata Giassi Udulutsch, Gabriel Mendes Marcusso, Gabriel Pavan Sabino, Vitor de Andrade Kamimura, Marco Antônio Assis: Contribution to critical revision, adding intellectual content 


\section{Conflicts of interest}

The authors declare that they have no conflict of interest related to the publication of this manuscript.

\section{References}

ARAUJO, D. \& ALVES, M. 2010. Climbing plants of a fragmented area of lowland Atlantic Forest, Igarassu, Pernambuco (Northeastern Brazil). Phytotaxa 8: 1-24.

APG. 2016. An update of the Angiosperm Phylogeny Group classification for the orders and families of flowering plants: APG IV. Botanical Journal of the Linnean Society 181 (1): 1-20.

BARROS, A.A.M.; RIBAS, L.A. \& ARAUJO, D.S.D. 2009. Trepadeiras do Parque Estadual da Serra da Tiririca, Rio de Janeiro, Brasil. Rodriguesia 60 (3): 681-694.

BARROSO, G.M.; MORIM, M.P.; PEIXOTO, A.L. \& ICHASO, C.L.F. 1999 Frutos e sementes - morfologia aplicada à sistemática de dicotiledôneas. Viçosa: Editora UFV. 444 pp.

BERTONI, J.E.A. \& MARTINS, F.R. 1987. Composição florística de uma floresta ripária na Reserva Estadual de Porto Ferreira, SP. Acta Botanica Brasilica 1 (1): $17-26$.

BERTONI, J.E.A.; TOLEDO FILHO, D.V.; LEITÃO FILHO, H.F.; FRANCO, G.A.D.C. \& AGUIAR, O.T. 2001. Flora arbórea e arbustiva do cerrado do Parque Estadual de Porto Ferreira (SP). Revista do Instituto Florestal 13 (2): 169-188.

BFG. 2015. Growing knowledge: an overview of Seed Plant diversity in Brazil. Rodriguésia 66 (4): 1085-1113.

BONNET, A.; CURCIO, G.R.; LAVORANTI, O.J. \& GALVÃO, F. 2011. Flora epifítica vascular em três unidades vegetacionais do Rio Tibagi, Paraná, Brasil. Rodriguésia 62: 491-498.

CARNEIRO, J.S. \& VIEIRA, A.O.S. 2012. Trepadeiras: florística da Estação Ecológica do Caiuá e chave de identificação vegetativa para espécies do Norte do Estado do Paraná. Acta Scientiarum Biological Sciences 34 (2): 217-223.

CARVALHO, P.G.; MELIS J.V.; MORELLATO, L.P. \& GROMBONE- GUARATINI, M.T. 2013. Critérios para a amostragem de lianas: comparação e estimativa da abundância e biomassa de lianas no cerrado. Revista Árvore 37 (6): 1037-1043.

CEPAGRI. Clima dos municípios paulistas. Disponível em: <http://www.cpa unicamp.br/outras-informacoes/clima_muni_457.html>. Acesso em: $31 \mathrm{de}$ janeiro de 2016.

COLLI, A.M.T.; SOUZA, S.A. \& SILVA, R.T. 2003. Pteridófitas do Parque Estadual de Porto Ferreira (SP), Brasil. Revista do Instituto Florestal 15 (1): 29-35.

DARWIN, C. 1867. On the moviments and habits of climbing plants. Journal of the Linnean Society, ser. Botany, 9: 1-118.

DEWALT, S.J.; SCHINITZER, S.A.; CHAVE, J.; BONGER, F.; BURNHAM, R.J.; CAI, Z.; CHUYONG, G.; CLARCK, D.B.; EWAANGO, C.E.N.; GERWING, J.J.; GORTAIRE, E.; HART, T.; IBARRA-MANRÍQUES, G.; ICKES, K.; KENFACK, D.; MACÍA, M.J.; MAKANA, J.; MARTÍNEZ-RAMOS, M.; MASCARO, J.; MOSES, S.; MULLER-LANDAU, H.C.; PARREN, M.P.E.; PARTHASARATHY, N.; PÉREZ-SALICRUP, D.R.; PUTZ, F.E.; ROMEROSALTOS, H. \& THOMAS, D. 2010. Annual rainfall and seasonality predict pan-tropical patterns of liana density and basal area. Biotropica 42 (3): 309-317.

DURIGAN, G.; RAMOS, V.S.; IVANAUSKAS, N. M.; FRANCO, G.A.D.C. 2012 Espécies indicadoras de fitofisionomias na transição Cerrado-Mata Atlântica no Estado de São Paulo. São Paulo: Secretaria do Meio Ambiente, 145p.

DURIGON, J.; CANTO-DOROW, T.S. \& EISINGER, S.M. 2009. Composição florística de trepadeiras ocorrentes em bordas de fragmentos de floresta estacional, Santa Maria, Rio Grande do Sul, Brasil. Rodriguesia 60 (2): 415-422.

DURIGON, J. \& WAECHTER, J.L. 2011. Floristic composition and biogeographic relations of a subtropical assemblage of climbing plants. Biodiversity Conservation 20 (5): 1027-1044

DURIGON, J., MIOTTO, S.T.S. \& GIANOLI, E. 2014. Distribution and traits of climbing plants in subtropical and temperate South America. Journal of Vegetation Science 25: 1484-1492.

ENGEL, V.L.; FONSECA, R.C.B. \& OLIVEIRA, R.E. 1998. Ecologia de lianas e o manejo de fragmentos florestais. Série Técnica IPEF 12 (32): 43-64.
FIDALGO, O. \& BONONI, V.L.R. 1984. Técnicas de coleta, preservação e herborização de material botânico. Instituto de Botânica, São Paulo. 62 p.

FILGUEIRAS, T.S.; NOGUEIRA, P.E.; BROCHADO, A.L. \& GUALALL, G.F 1994. Caminhamento: um método expedito para levantamentos florísticos qualitativos. Cadernos de Geociências 12: 39- 347.

FORZZA, R.C.; PIFANO, D.S.; OLIVEIRA-FILHO, A.R.; MEIRELES, L.D.; FARIA, P.L.; SALIMENA, F.R.; MYNSSEN, C.M. \& PRADO, J. 2014. Flora vascular da Reserva Biológica da Represa do Grama, Minas Gerais, e sua relação florística com outras florestas do sudeste brasileiro. Rodriguésia 65 (2): 275-292.

GALLAGHER, R. V.; LEISHMAM, M. R. \& MOLES, A. T. 2011. Traits and ecological strategies of Australian tropical and temperate climbing plants. Jounal of Biogegraphy 38 (5): 828-839.

GENTRY, A. H. 1979. Additional generic mergers in Bignoniaceae. Annals of the Missouri Botanical Garden 66: 778-787.

GENTRY, A.H. \& DODSON, C. 1987. Contribution of Nontrees to Species Richness of a Tropical Rain Forest. Biotropica 19 (2): 149-156.

GENTRY, A.H. 1991. The distribution and evolution of climbing plants, pp. 3-49, in: Putz, F.E. and Mooney, H.A. (ed.). The biology of vines. Cambridge, Cambridge University Press.

GERWING, J.J.; SCHNITZER, S.A.; BURNHAM, R.J.; BONGERS, F.; CHAVE, J.; DEWALT, S.; EWANGO, C.E.N.; FOSTER, R.; KENFACK, D.; MARTÍNEZRAMOS, M.; PARREN, M.; PARTHASARATHY, N.; PÉREZ-SALICRUP, D.; PUTZ, F.E. \& THOMAS, D.W. 2006. A Standard Protocol for Liana Censuses. Biotropica 38 (2): 256-261

GIANOLI, E. Evolutionary implications of the climbing habit in plants. 2015. In: Schnitzer, S.A.; Bongers, F.; Burnham, R.J. \& Putz, F.E. Ecology of lianas, Wiley Blackwell, Oxford. P.p. 239-250.

GIANOLI, E.; TORRES-DIAZ, C.; RUIZ, E., SALGADO-LUARTE, C.; MOLINAMONTENEGRO, M.A.; SALDAÑA, A. \& RIOS, R.S. 2016. Woody climbers show greater population genetic differentiation than trees: Insights into the link between ecological traits and diversification. Evolution 70 (12): 2736-2745.

HERGATY, E.E. 1991. Vine-host interactions, pp. 357-375, in: Putz, F.E. and Mooney, H.A. (ed.). The biology of vines. Cambridge, Cambridge University Press.

HORA, R.C. \& SOARES, J.J. 2002. Estrutura fitossociológica da comunidade de lianas em uma floresta estacional na Fazenda Cachim, São Carlos, SP. Revista Brasileira de Botânica 25 (3): 323-329.

IBARRA-MANRÍQUEZ, G.; RENDÓN-SANDOVAL, F.J.; CORNEJO-TENORIO, G. \& CARRILLO-REYES, P.2013. Lianas of Mexico. Botanical Sciences 9 (3): $365-417$.

IBGE. Instituto Brasileiro de Geografia e Estatística. 2012. Manual técnico da vegetação brasileira. Instituto Brasileiro de Geografia e Estatística. Rio de Janeiro. $276 \mathrm{p}$.

KARK, S. \& VAN RENSBURG, B.J. 2006. Ecotones: marginal or central areas of transition? Israel Journal of Ecology and Evolution 52 (1): 29-53.

KARK, S. 2012. Ecotones and Ecological Gradients, pp. 147-160, in: Meyers, R.A (ed.). Encyclopedia of Sustainability Science and Technology. New York: Springer Science + Business Media.

KENT, M.; GILL, W.J.; WEAVER; R.E. \& ARMITAGE, R.P. 1997. Landscape and plant community boundaries in biogeography. Progress in Physical Geopgraphy 21 (3): 315-353.

KERSTEN, R.A. 2010. Epífitas vasculares - histórico, participação taxonômica e aspectos relevantes, com ênfase na Mata Atlântica. Hoehnea 37: 9-38.

LADWIG, L. \& MEINERS, S. 2010. Spatiotemporal dynamics of lianas during 50 years of succession to temperate forest, Ecology 91 (3): 671-680.

LETCHER, S.G. \& CHAZDON, R.L. 200. Lianas and self-supporting plants during tropical forest succession. Forest Ecology and Management 257 (10): 2150-2156.

LOHMANN, L. 2006. Untangling the phylogeny of Neotropical lianas (Bignonieae, Bignoniaceae). American Journal of Botany 93 (4): 304-318.

LOHMANN, L.G. 2015. Bignoniaceae in Lista de Espécies da Flora do Brasil. Jardim Botânico do Rio de Janeiro. Disponível em: http://floradobrasil.jbrj.gov. br/jabot/floradobrasil/FB112305. Acesso em: 31 de janeiro de 2017.

LOMBARDI, J.A.; TEMPONI, L.G. \& LEITE C.A. 1999. Mortality and diameter growth of lianas in a semideciduos forest fragmente in southeastern Brazil. Acta Botanica Brasilica 13 (2): 159-165. 
MAMEDE, M.C.H.; SEBASTIANI, R.; ALMEIDA, R.F.; FRANCENER, A. \& AMORIM, A.M.A. 2015. Malpighiaceae in Lista de Espécies da Flora do Brasil. Jardim Botânico do Rio de Janeiro. Disponível em: http://floradobrasil.jbrj.gov. br/jabot/floradobrasil/FB155. Acesso em: 31 de janeiro de 2017.

MANTOVANI, W. \& MARTINS, F.R. 1993. Florística do cerrado na Reserva Biológica de Moji Guaçu, estado de São Paulo. Acta Botânica Brasílica 7 (1): 33-60.

MARCUSSO, G.M.; DICKFELDT, E.P.; BERTONI, J.E.A. \& MONTEIRO, R. 2016. Epífitas vasculares do Parque Estadual de Porto Ferreira, São Paulo, Brasil. Revista do Instituto Florestal 28 (2): 119-133.

MARCUSSO, G.M. \& MONTEIRO, R. 2016. Composição florística das epífitas vasculares em duas fisionomias vegetais no município de Botucatu, estado de São Paulo, Brasil. Rodriguésia, 67 (3): 553-569.

MORELLATO, P.C. \& LEITÃO FILHO, H.F. 1996. Reproductive phenology of climbers is Southeastern Brazilian forest. Biotropica 28 (2): 180-191.

MORELLATO, P.C. \& LEITÃO FILHO, H.F. 1998. Levantamento florístico da comunidade de trepadeiras de uma floresta semidecídua no Sudeste do Brasil. Boletim do Museu Nacional, Nova Série Botânica 103: 1-15.

NABE-NIELSEN, J. 2001. Diversity and distribution of lianas in a Neotropical rain forest, Yasuní National Park, Ecuador. Journal of Tropical Ecology 17: 1-19.

ODUM, E.P. 1969. Ecologia. Interamericana, México. 412p.

OLIVEIRA, A.P.C. 2012. Flora vascular não-arbórea do Parque Estadual de Porto Ferreira, SP, Brasil. Dissertação de Mestrado. Universidade Estadual Paulista "Júlio Mesquita Filho", Rio Claro.

OLIVEIRA, E.A.; MARIMON, B.S.; FELDPAUSCH, T.R.; COLLIF, G.R.; MARIMON-JUNIOR, B.H.; LLOYD, J.; LENZA, E.; MARACAHIPES, L.; OLIVEIRA-SANTOS, C. \& PHILLIPS, O.L. 2014. Diversity, abundance and distribution of lianas of the Cerrado-Amazonian forest transition, Brazil. Plant Ecology and Diversity 7 (1-2): 231-240.

OSACO, M. 2012. Florística e fitossociologia do estrato arbustivo de área de transição savana-floresta no Parque Estadual de Porto Ferreira. Dissertação de Mestrado. Universidade Estadual Paulista "Júlio de Mesquita Filho", Rio Claro.

PERES, M.K. 2016. Estratégias de dispersão de sementes no Bioma Cerrado: considerações ecológicas e filogenéticas. Tese de Doutorado, Brasília, Universidade de Brasília.

PÉREZ-SALICRUP, D.R.; SORK, V.L. \& PUTZ, F.E. 2001. Lianas and trees in a Lianas Forest of Amazonian Bolivia. Biotropica 33 (1): 34-47.

PHILLIPS, O.L.; VÁSQUEZ, M.R.; ARROYO, L.; BAKER, T.; KILLEEN, T.; LEWIS, S.L.; YANDVINDER, M.; MENDONZA, A.M.; NEIL, D.; VARGAS, P.C.; ALEXIADES, M.; CERÓN, C.; DI FIORE, A.; ERWIN, T.; JARDIM, A.; PALACIOS, W.; SALDIAS, M. \& VINCETI, B. 2002. Increasing dominance of large lianas in Amazonian forests. Nature 418: 770-774.

PINHEIRO, M.H.O. \& MONTEIRO, R. 2008. Florística de uma Floresta Estacional Semidecidual, localizada em ecótono savânico-florestal, no município de Bauru, SP, Brasil. Acta Botanica Brasilica, 22 (4): 1085-1094.

PUTZ, F.E. \& CHAI, P. 1987. Ecological studies of lianas in Lambir National Park, Sarawak. Journal of Ecology 75 (2): 523-531.

REZENDE, A.A. \& RANGA, N.T. 2005. Lianas da Estação Ecológica do Noroeste Paulista, São José do Rio Preto/Mirassol, SP, Brasil. Acta Botânica Brasílica 19 (2): 273-279

REZENDE, A.A.; RANGA, N.T. \& PEREIRA, R.A.S. 2007. Lianas de uma floresta estacional semidecidual, município de Paulo de Faria, norte do estado de São Paulo, Brasil. Revista Brasileira de Botânica 30 (3): 451-461.

São Paulo. 2003. Instituto Florestal. Plano de Manejo do Parque Estadual de Porto Ferreira

SANTOS, K.; KINOSHITA, L.S. \& REZENDE, A.A. 2009. Species composition of climbers in seasonal semideciduous forest fragments of Southeastern Brazil. Biota Neotropica 9: 175-188.

SABINO, G.P. 2013. Florística e Fitossociologia de uma comunidade arbórea em contato savana-floresta no Parque Estadual de Porto Ferreira, Porto Ferreira, São Paulo. Trabalho de Conclusão de Curso (Graduação em Ciências Biológicas) -Universidade Estadual Paulista "Júlio Mesquita Filhos", Rio Claro.

SCHNITZER, S.A. \& BONGERS, F. 2002. The ecology of lianas and their role in forest. Trends in Ecology \& Evolution 17 (5): 223-230.
SCHNITZER, S.A. \& BONGERS, F. 2011. Increasing liana abundance and biomass in tropical forests: emerging patterns and putative mechanisms. Ecology Letters 14 (4): 397-402.

SCHNITZER, S.A.; VAN DER HEIJDEN, G.M.F. \& POWERS, J.S. 2016. Addressing the challenges of including lianas in global vegetation models. Proceedings of the National Academy of Sciences of the United States of America 113 (1): 5-6.

SOMNER, G.V.; FERRUCCI, M.S.; ACEVEDO-RODRÍGUEZ, P.; PERDIZ, R.O.; COELHO, R.L.G. \& MEDEIROS, H. 2015. Sapindaceae in Lista de Espécies da Flora do Brasil. Jardim Botânico do Rio de Janeiro. Disponível em: http://floradobrasil.jbrj.gov.br/jabot/floradobrasil/FB216. Acesso em: 31 de janeiro de 2017.

TIBIRIÇÁ, Y.J.A.: COELHO, L.F.M. \& MOURA, L.C. 2006. Florística de lianas em um fragmento de floresta estacional semidecidual, Parque Estadual de Vassununga, Santa Rita do Passa Quatro, SP, Brasil. Acta Botanica Brasilica 20 (2): 339-346.

UDULUTSCH, R.G.; ASSIS, M.A. \& PICCHI, D.G. 2004. Florística de trepadeiras numa floresta estacional semidecídua, Rio Claro-Araras, estado de São Paulo, Brasil. Revista Brasileira de Botânica 27 (1): 125-134.

UDULUTSCH, R.G.; SOUZA, V.C.; RODRIGUES, R.R. \& DIAS, P. 2010. Composição florística e chaves de identificação para as lianas da Estação Ecológica dos Caetetus, estado de São Paulo, Brasil. Rodriguésia 61 (4): 715-730.

VAN DER HEIDJEN, G.M.; SCHNITZER, S.A.; POWERS, J.S. \& PHILLIPS, O.L. 2013. Liana Impacts on Carbon Cycling, Storage and Sequestration in Tropical Forests. Biotropica 45(6): 682-692.

VAN DER PIJL, L. 1982. Principles of dispersal in higher plants. 3rd ed. Springer Verlag, New York. 139p.

VARGAS, B.C.; ARAÚJO, G.M.; SCHIAVINI, I.; ROSA, P.O. \& HATTORI, E.K.O. 2013. Florística de trepadeiras em floresta semidecidual e em Mata ciliar no vale do Rio Araguari, MG. Bioscience Journal 29 (1): 185-197.

VARGAS, B.C. \& ARAÚJO, G.M. 2014. Florística de trepadeiras em fragmentos de florestas semideciduais em Uberlândia, Minas Gerais, Brasil. Rodriguésia 65 (1): $49-59$.

VILLAGRA, B.L.P. \& ROMANIUC NETO, S.R. 2010. Florística de trepadeiras no Parque Estadual das Fontes do Ipiranga, São Paulo, SP, Brasil. Revista Brasileira de Biociências 8 (2): 186-200.

VILLAGRA, B.L.P. \& ROMANIUC NETO, S.R. 2014. Nomenclatura das plantas de hábito trepador, pp. 1-12, in: B.L.P., Villagra, M.M.R.F., Melo, S., Romaniuc-Neto, L.M. \& Barbosa, L.M. (eds). Diversidade e conservação de trepadeiras: contribuição para a restauração de ecossistemas brasileiros. São Paulo: Instituto de Botânica.

VIVEK, P. \& N. PARTHASARATHY. 2015. Liana community and functional trait analysis in tropical dry evergreen forest of India. Journal of Plant Ecology 8 (5): 501-512.

YORKE, S.R.; SCHNITZER, S.A.; MASCARO, J.; LECHTER, S.G. \& CARSON, W.P. 2013. Increasing liana abundance and basal area in a tropical forest: the contribution of long-distance clonal colonization. Biotropica 45 (3): 317-324.

WEISER, V. de L. \& GODOY, S.A.P. 2001. Florística em um hectare de cerrado sensu stricto na ARIE - Cerrado Pé de Gigante, Santa Rita do Passa Quatro, SP. Acta Botanica Brasilica 15 (2): 201-212.

WEISER, V. de L. 2002. Ecologia e sistemática de lianas em um hectare de cerrado stricto sensu da ARIE- Cerrado Pé-de Gigante, Santa Rita do Passa Quatro, SP. Dissertação de Mestrado, Ribeirão Preto, Universidade de São Paulo.

WEISER, V. de L. 2007. Árvores, arbustos e trepadeiras do cerradão do Jardim Botânico Municipal de Bauru, SP. Tese de Doutorado, Campinas, Universidade de Campinas.

WEISER, V. de L. \& MARTINS, F.R. 2014. Trepadeiras do cerrado paulista, pp. 58-71, in: B.L.P., Villagra, M.M.R.F., Melo, S., Romaniuc-Neto, L.M. \& Barbosa, L.M. (eds). Diversidade e conservação de trepadeiras: contribuição para a restauração de ecossistemas brasileiros. São Paulo: Instituto de Botânica.

Received: 28/03/2017

Revised: 17/07/2017

Accepted: $24 / 01 / 2018$

Published online: 12/03/2018 